\title{
Lateralized Effects of Unilateral Thalamotomy and Thalamic Stimulation in Patients with Essential Tremor
}

\author{
Mi J. Kim ${ }^{\mathrm{a}}$ \\ Sang R. Jeon ${ }^{\mathrm{b}}$ \\ Sung R. Kim ${ }^{\mathrm{a}}$ \\ Myoung C. Lee ${ }^{a}$ \\ Sun J. Chung a

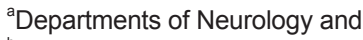 \\ ${ }^{b}$ Neurological Surgery, \\ Asan Medical Center, \\ University of Ulsan College of Medicine, \\ Seoul, Korea
}

\begin{abstract}
Background and Purpose: Stereotactic thalamotomy has been an effective surgical procedure in the treatment of medically refractory essential tremor (ET), however, little is known about the bilateral effects of unilateral ventralis intermedius (Vim) thalamotomy and Vim deep brain stimulation (DBS). We studied the lateralized effects of unilateral Vim thalamotomy and Vim DBS in ET patients. Methods: Vim thalamotomy was performed in 6 patients and Vim DBS in 6. Patients were evaluated preoperatively and at 3 and 6 months postoperatively using the Clinical Rating Scale for Tremor (CRST). Results: The contralateral Part A (tremor localization/severity rating) and Part B (specific motor tasks/function rating) subscores, and axial subscores of CRST significantly improved after unilateral Vim thalamotomy or Vim DBS. On the side ipsilateral to surgery, ET patients demonstrated no significant improvements in the Part A and Part B subscores of CRST. The Part C (functional disabilities resulting from tremor) subscores and total scores of CRST were significantly improved after surgery. Conclusions: Vim thalamotomy and DBS may be equally effective for the management of contralateral and axial tremor in ET patients, but both interventions may not improve tremor on the side ipsilateral to surgery.

Journal of Movement Disorders 2011;4:64-67
\end{abstract}

Key Words: Essential tremor, Thalamotomy, Deep brain stimulation.

Essential tremor (ET) is a common movement disorder, characterized by postural and kinetic tremors typically involving the hands, forearms, and head with an oscillation frequency ranging from 4 to $12 \mathrm{~Hz}$. ET may be treated pharmacologically, but patients with ET may have variable pharmacological responsiveness. In addition, tremor amplitude and subsequent disability increase with age. ${ }^{1}$ Patients may experience functional decline and loss of ability to perform some activities of daily living independently, thus requiring surgical management.

Stereotactic thalamotomy has been an effective surgical procedure in the treatment of medically refractory ET, ${ }^{2,3}$ Recently, deep brain stimulation (DBS) of thalamic nuclei has become more widespread, due to the reversibility of its effects and its reduced complication profile compared with thalamotomy. ${ }^{4,5}$

ET is characterized by bilateral tremor between the upper extremities. Older patients and patients with marked asymmetric tremor may benefit from unilateral surgery. Although unilateral thalamic DBS has shown substantial benefits, ${ }^{6,7}$ little is known about the bilateral effects of unilateral ventralis intermedius (Vim) thalamotomy and Vim DBS. We report here results from a series of $12 \mathrm{ET}$ patients managed in our center with unilateral surgical treatment.

\section{Methods}

\section{Patients}

Between June 2005 and March 2007, 12 consecutive patients underwent unilateral stereotactic surgery for the management of ET at Asan Medical Center. ET diagnosis was based on the guidelines of the Tremor Investigational Group. ${ }^{8}$ Selection criteria included clinically diagnosed ET with bilateral hand tremor, severe tremor despite optimal adjustment of medication, normal brain MRI, no surgical contraindications, and no dementia or major ongo- 
ing psychiatric illness.

Patients were excluded if they scored $<24$ in the Mini Mental State Examination or $>18$ in the Beck Depression Inventory. ${ }^{9}$ Medications remained the same for 30 days before surgery and for the first 3 months after surgery.

\section{Surgery}

Stereotactic surgery using the Leksell G frame was performed under local anesthesia by a single neurosurgeon (S.R.J) one day after withdrawal of medications. Patients underwent unilateral electrode implantation for DBS or unilateral thalamotomy according to patient preference, without randomization between the two treatment modalities. The Vim thalamic nucleus contralateral to the dominant hand was located by MRI, intra-operative electrophysiological recordings and macroelectrode stimulation. The initial target was $13-15 \mathrm{~mm}$ lateral to the AC-PC midline, posterior one fourth of the inter-commissural line, and $0 \mathrm{~mm}$ below the inter-commissural line.

For DBS, quadrupolar electrodes (model 3387, Medtronic Inc.) were implanted and connected to a subcutaneous programmable pulse generator (Soletra TM; Medtronic) under general anesthesia. For thalamotomy, a permanent lesion was produced by radiofrequency applying $3 \mathrm{~mm}$ bare tip of a $1.5 \mathrm{~mm}$ diameter electrode, heated at a temperature of $70^{\circ} \mathrm{C}$ for $70 \mathrm{sec}-$ onds after a temporary lesion $\left(40^{\circ} \mathrm{C}\right.$ for 40 seconds) to confirm safety. Second lesion was made $2 \mathrm{~mm}$ superiorly as the electrode was withdrawn. Postoperative brain CT was used to assess surgical complications.

\section{Clinical assessments}

Patients were evaluated preoperatively and at 3 and 6 months postoperatively using the Clinical Rating Scale for Tremor (CRST). ${ }^{10}$ The CRST consists of three parts: Part A (tremor localization/severity rating), Part B (specific motor tasks/function rating), ant Part $\mathrm{C}$ (functional disabilities resulting from trem- or). Part A (scores 1-10) quantifies the tremor at rest, with posture holding, and with action and intention maneuvers, for nine parts of the body and orthostatic tremor. Part B (scores 11-15) relates to action tremors of the upper extremities, particularly writing and pouring liquids. Part $\mathrm{C}$ assesses functional disability, including the evaluation of the severity of tremor with speaking, eating, bringing liquids to the mouth, hygienic care, dressing, and working. The 'axial' subscore was defined as the sum of the scores for the face, tongue, voice, head, and trunk (items 1-4 and 7).

Patients were evaluated by one rater (M.J.K.), with each patient always evaluated by the same rater. Nine patients were evaluated at 3 months postoperatively and 10 patients at 6 months.

\section{Statistical analysis}

The primary outcome measures were the differences in CRST scores according to body side, including ipsilateral, contralateral, and axial subscores, between baseline and 3 and 6 months after surgery. The secondary outcome measures were the differences in CRST scores between the thalamotomy and DBS patients. Data were analyzed using Student's $t$-test, Wilcoxon signed rank test, or Mann-Whitney U tests, where indicated. Statistical Package for the Social Sciences (SPSS) for Windows (version 12, SPSS Inc.) was used for all statistical analysis and $p$ values $<0.05$ were regarded as statistically significant.

\section{Results}

The clinical characteristics of the patients at baseline are summarized in Table 1. Mean age at surgery was $70.9 \pm 4.7$ years (range, 66-81 years) and mean duration of disease was $19.6 \pm 13.4$ years. Mean Part A subscore of CRST was 16.1, Part B subscore was 24.9, and Part C subscore was 17.8. The left-sided Vim thalamic nucleus was targeted in all patients.

Table 1. Baseline characteristics of the patients with essential tremor

\begin{tabular}{|c|c|c|c|c|}
\hline \multirow{2}{*}{ Characteristics } & \multirow{2}{*}{ Patients with ET $(n=12)$} & \multicolumn{2}{|c|}{ Stereotactic surgery } & \multirow{2}{*}{$p$ value } \\
\hline & & $\operatorname{Vim} \operatorname{DBS}(n=6)$ & Vim thalamotomy $(n=6)$ & \\
\hline \multicolumn{5}{|l|}{ Clinical variables } \\
\hline Age at surgery (yr) & $70.9 \pm 4.7$ & $70.2 \pm 2.7$ & $71.6 \pm 6.3$ & 0.608 \\
\hline Duration of disease (yr) & $19.6 \pm 13.4$ & $21.7 \pm 10.7$ & $17.5 \pm 16.4$ & 0.614 \\
\hline Male & $10(83.3 \%)$ & $4(66.7 \%)$ & $6(100 \%)$ & 0.145 \\
\hline Part $\mathrm{A}^{\dagger}$ & $16.2 \pm 6.2$ & $15.7 \pm 2.8$ & $16.5 \pm 5.9$ & 0.828 \\
\hline Part $B^{\ddagger}$ & $24.9 \pm 7.5$ & $24.2 \pm 7.9$ & $25.7 \pm 7.7$ & 0.748 \\
\hline Part C§ & $17.8 \pm 6.1$ & $16.8 \pm 7.3$ & $18.7 \pm 5.1$ & 0.627 \\
\hline Axial scores & $2.3 \pm 2.2$ & $2.2 \pm 2.6$ & $2.3 \pm 2.1$ & 0.905 \\
\hline Total scores & $58.8 \pm 18.4$ & $56.7 \pm 21.1$ & $60.8 \pm 6.9$ & 0.714 \\
\hline
\end{tabular}

Values shown are mean \pm standard deviation. *tremor severity assessed according to the Clinical Rating Scale for Tremor, 10 tremor localization/severity rating, ‡specific motor tasks/function rating, sfunctional disabilities resulting from tremor. ET: essential tremor, Vim: ventral intermediate thalamic nucleus, DBS: deep brain stimulation. 
Table 2. Side-specific efficacy of unilateral Vim thalamotomy and Vim DBS on tremor in patients with essential tremor

\begin{tabular}{|c|c|c|c|c|c|}
\hline \multirow{2}{*}{ Tremor subscores* } & \multirow{2}{*}{$\begin{array}{c}\text { Baseline } \\
(n=12)\end{array}$} & \multicolumn{2}{|c|}{ Postoperative state } & \multicolumn{2}{|c|}{$p$ value } \\
\hline & & 3 months $(n=9)$ & 6 months $(n=10)$ & 3 months vs. baseline & 6 months vs. baseline \\
\hline \multicolumn{6}{|l|}{ Contralateral scores } \\
\hline Part $\mathrm{A}^{\dagger}$ & $7.7 \pm 2.5$ & $1.6 \pm 1.1$ & $2.4 \pm 1.9$ & 0.008 & 0.004 \\
\hline Part $B^{\ddagger}$ & $11.4 \pm 3.2$ & $3.1 \pm 2.0$ & $4.0 \pm 3.2$ & 0.008 & 0.004 \\
\hline \multicolumn{6}{|l|}{ Ipsilateral scores } \\
\hline Part $A^{\dagger}$ & $6.2 \pm 2.9$ & $6.3 \pm 2.8$ & $5.9 \pm 2.1$ & $>0.999$ & 0.531 \\
\hline Part $B^{\ddagger}$ & $10.2 \pm 4.5$ & $9.6 \pm 3.9$ & $10.3 \pm 3.4$ & 0.688 & 0.688 \\
\hline Axial scores & $2.3 \pm 2.2$ & $1.1 \pm 0.8$ & $1.0 \pm 1.6$ & 0.066 & 0.017 \\
\hline Part $C^{\S}$ & $17.8 \pm 6.1$ & $4.0 \pm 3.7$ & $6.3 \pm 5.6$ & 0.008 & 0.004 \\
\hline Total scores & $58.8 \pm 8.4$ & $26.6 \pm 10.7$ & $30.9 \pm 12.6$ & 0.008 & 0.008 \\
\hline
\end{tabular}

Data are presented as mean \pm standard deviation. ${ }^{*}$ tremor severity assessed according to the Clinical Rating Scale for Tremor, $10+$ tremor localization/severity rating, „specific motor tasks/function rating, sfunctional disabilities resulting from tremor. Vim: ventral intermediate thalamic nucleus, DBS: deep brain stimulation.

Six patients underwent Vim thalamotomy and 6 underwent Vim DBS; there were no differences in baseline characteristics between these two groups of patients.

\section{Effect of unilateral Vim stereotactic surgery}

Baseline and postoperative tremor scores are shown in Table 2. Following unilateral Vim stereotactic surgery, the Part A subscore of body parts contralateral to the surgery improved $76.25 \%(p=0.008)$ at 3 months and $67.06 \%(p=0.004)$ at 6 months. The Part B subscore of body parts contralateral to the surgery improved $84.5 \%(p=0.008)$ at 3 months and $77.43 \%$ ( $p=0.004)$ at 6 months. Between 3 and 6 months, the subscores of Part A and B increased slightly, but the differences were not statistically significant.

Compared with baseline, the Part A and B subscores of body parts ipsilateral to Vim stereotactic surgery were not improved 3 and 6 months after surgery (Table 2). Axial subscores improved $27.67 \%(p=0.066)$ at 3 months and $65.77 \%(p=$ $0.017)$ at 6 months.

In comparison with baseline score, the Part $\mathrm{C}$ subscore improved 78.94\% $(p=0.008)$ at 3 months and $67.96 \%(p=0.004)$ at 6 months. Total tremor score improved $53.75 \%(p=0.008)$ at 3 months and $48.49 \%(p=0.008)$ at 6 months.

Six months after surgery, there were no differences in tremor scores, including Parts A, B, and C subscores, axial subscores, and total tremor scores, between patients who underwent Vim thalamotomy and those who underwent Vim DBS.

\section{Adverse effects}

One patient who underwent Vim DBS had a small amount of intracerebral hemorrhage (ICH) along the tract of lead implantation, but this ICH was asymptomatic. One patient who underwent Vim thalamotomy had transient dysarthria, which disappeared 10 days after surgery. Subcutaneous connector adhesion at the supraclavicular area was found in one patient who underwent Vim DBS.

\section{Discussion}

We found that unilateral Vim thalamotomy and DBS were associated with significant postoperative improvements in tremor of body parts contralateral to the targeted Vim and on tremor of axial body parts. However, neither procedure had a significant therapeutic effect on the tremor of body parts ipsilateral to the targeted Vim. These results are in agreement with a recent report, showing that bilateral effects of unilateral thalamic DBS were not observed in 8 patients with ET, but were shown in one patient with mitochondrial encephalopathy. ${ }^{11}$ In contrast, others have reported mild improvements in some measures of ET ipsilateral to the unilateral Vim DBS implantation site, suggesting that the observed ipsilateral improvement largely resulted from reduced mechanical outflow from the target side, rather than from a direct effect on the brain. ${ }^{12}$ In that study, however, the ipsilateral effect of Vim DBS was clinically insignificant and the follow-up period was approximately 3 months. ${ }^{12}$ Interestingly, we found that unilateral Vim thalamotomy and DBS had a significant effect on tremor of axial body parts, a measurement not analyzed in previous studies. This partial improvement may be due to the bilateral innervations of axial muscles.

We previously reported that, unilateral subthalamic nucleus (STN) DBS showed bilateral effects in patients with Parkinson's disease (PD) ${ }^{13}$ suggesting two possible pathogenic mechanisms: 1) bilateral projections from the supplementary motor area to both striata; 2) or bilateral projections of the globus pallidus pars internus, which receives input from the STN, to both thalami or to the brainstem. However, in agreement with the findings presented here, we found that the ipsilateral effect of unilateral STN DBS was predominant in the subscore of ipsilateral bradykinesia, whereas the subscore of ipsilateral tremor was not significantly improved. ${ }^{13}$ These results suggest that the pathogenic mechanisms and anatomical basis of tremor may differ between tremor and bradykinesia in PD. Although the pathogenic mechanism of tremor differs between 
PD and ET, the Vim thalamic nucleus plays a critical role in modulating the activity of the cortico-striato-pallido-thalamocortical pathways in PD and the cerebellothalamocortical pathways in ET, and production of tremor in both diseases. Thus, Vim stereotactic surgery is highly effective in treating contralateral tremor in both diseases. The absence of an ipsilateral effect of unilateral Vim thalamotomy and DBS in ET patients may be related to the independent neural network alteration between right and left hemispheres in the production of tremor, and absence of bilateral modulation by Vim stereotactic surgery.

We observed no differences in the side-specific effects of surgery on tremor between unilateral Vim thalamotomy and DBS. A previous study, including 6 ET patients who received thalamotomy and 7 ET patients who underwent thalamic DBS, showed that two surgical interventions were equally effective in the suppression of drug-resistant tremor. ${ }^{14}$ That stu$\mathrm{dy}$, however, did not evaluate the effects of surgery on the ipsilateral and axial tremors. Although the precise mechanism of DBS action is unclear, a number of experimental studies have suggested that local inhibition is the major effect of this intervention. ${ }^{15-17}$ In contrast, Vim DBS may facilitate rather than block the cerebellothalamocortical pathway in patients with ET. ${ }^{18-20}$ Given these dual effects of DBS, differences in side-specific effects between Vim thalamotomy and Vim DBS might be expected, but they were clinically insignificant in our patients.

The present study had several limitations. First, the tremor scale rating was evaluated only by neurological examination without objective electrophysiological studies. Second, the number of subjects was small and the follow-up duration was short. Third, the ET patients were not randomized to the two surgical interventions.

In conclusion, unilateral Vim thalamotomy and DBS did not show significant therapeutic effects on ipsilateral tremor in ET patients, but had partial effects on axial tremor and marked effect on contralateral tremor. Larger, well-designed longerterm studies are needed to confirm the significance of our findings.

\section{REFERENCES}

1. Elble RJ. Essential tremor frequency decreases with time. Neurology 2000;55:1547-1551.

2. Goldman MS, Kelly PJ. Stereotactic thalamotomy for medically intractable essential tremor. Stereotact Funct Neurosurg 1992;58:22-25.

3. Zirh A, Reich SG, Dougherty PM, Lenz FA. Stereotactic thalamotomy in the treatment of essential tremor of the upper extremity: reassessment including a blinded measure of outcome. J Neurol Neurosurg Psychiatry 1999;66:772-775.

4. Benabid AL, Pollak P, Gao D, Hoffmann D, Limousin P, Gay E, et al. Chronic electrical stimulation of the ventralis intermedius nucleus of the thalamus as a treatment of movement disorders. J Neurosurg 1996; 84:203-214.

5. Koller WC, Lyons KE, Wilkinson SB, Troster AI, Pahwa R. Long-term safety and efficacy of unilateral deep brain stimulation of the thalamus in essential tremor. Mov Disord 2001;16:464-468.

6. Koller WC, Lyons KE, Wilkinson SB, Pahwa R. Efficacy of unilateral deep brain stimulation of the VIM nucleus of the thalamus for essential head tremor. Mov Disord 1999; 14:847-850.

7. Ondo W, Almaguer M, Jankovic J, Simpson RK. Thalamic deep brain stimulation: comparison between unilateral and bilateral placement. Arch Neurol 2001;58:218-222.

8. Findley LJ, Koller WC. Definitions and behavioral classifications. In: Findley LJ, Koller WC. Handbook of tremor disorders. New York: Marcel Dekker, 1995:1-5.

9. Beck AT, Ward CH, Mendelson M, Mock J, Erbauch J. An inventory for measuring depression. Arch Gen Psychiatry 1961;4:561-571.

10. Fahn S, Tolosa E, Marin C. Clinical rating scale for tremor. In: Jankovic J, Tolosa E. Parkinson's Disease and Movement Disorders. Baltimore: Williams \& Wilkins, 1993:271-280.

11. Kovacs N, Pal E, Merkli H, Kellenyi L, Nagy F, Janszky J, et al. Bilateral effects of unilateral thalamic deep brain stimulation: a case report. Mov Disord 2008;23:276-279.

12. Ondo W, Dat Vuong K, Almaguer M, Jankovic J, Simpson RK. Thalamic deep brain stimulation: effects on the nontarget limbs. Mov Disord 2001;16:1137-1142.

13. Chung SJ, Jeon SR, Kim SR, Sung YH, Lee MC. Bilateral effects of unilateral subthalamic nucleus deep brain stimulation in advanced Parkinson's disease. Eur Neurol 2006;56:127-132.

14. Schuurman PR, Bosch DA, Bossuyt PM, Bonsel GJ, van Someren EJ, de Bie RM, et al. A comparison of continuous thalamic stimulation and thalamotomy for suppression of severe tremor. N Engl J Med 2000; 342:461-468.

15. Filali M, Hutchison WD, Palter VN, Lozano AM, Dostrovsky JO. Stimulation-induced inhibition of neuronal firing in human subthalamic nucleus. Exp Brain Res 2004;156:274-281.

16. Lozano AM, Mahant N. Deep brain stimulation surgery for Parkinson's disease: mechanisms and consequences. Parkinsonism Relat Disord 2004;10:S49-S57.

17. McIntyre CC, Savasta M, Kerkerian-Le Goff L, Vitek JL. Uncovering the mechanism(s) of action of deep brain stimulation: activation, inhibition, or both. Clin Neurophysiol 2004;115:1239-1248.

18. Molnar GF, Sailer A, Gunraj CA, Lang AE, Lozano AM, Chen R. Thalamic deep brain stimulation activates the cerebellothalamocortical pathway. Neurology 2004;63:907-909.

19. Perlmutter JS, Mink JW, Bastian AJ, Zackowski K, Hershey T, Miyawaki E, et al. Blood flow responses to deep brain stimulation of thalamus. Neurology 2002;58:1388-1394.

20. Molnar GF, Sailer A, Gunraj CA, Cunic DI, Lang AE, Lozano AM, et al. Changes in cortical excitability with thalamic deep brain stimulation. Neurology 2005;64:1913-1919. 